\title{
Modified Wilson Technique for Treatment of Hallux Valgus
}

\author{
Ali Zein A. A. Al-Khooly*, Mohamed Y. Hasan, Hesham A. Mohamed \\ Department of Orthopedic and Trauma, El-Minia University Hospital, El-Minia, Egypt \\ Email: ${ }^{*}$ profalizein@yahoo.com
}

Received 22 January 2014; revised 26 February 2014; accepted 7 Mrach 2014

Copyright (C) 2014 by authors and Scientific Research Publishing Inc.

This work is licensed under the Creative Commons Attribution International License (CC BY). http://creativecommons.org/licenses/by/4.0/

(c) (i) Open Access

\begin{abstract}
Background: Moderate to severe hallux valgus deformity demands correction of the deformity. The purpose of the current study was to evaluate the results and advantages of the modified Wilson technique for treatment of hallux valgus. Twenty four operations of distal oblique osteotomies on the first metatarsal bone as described by Wilson (1963) with some modifications were performed on fifteen patients suffering from hallux valgus, done in the Department of Orthopedic Surgery and Traumatology of El-Minia University Hospital; nine of them were bilateral and six were unilateral. The osteotomy site was fixed by $\mathrm{K}$. wire after displacement of the distal fragment laterally and proximally. A below the knee plaster cast was applied and the patients were encouraged to bear weight partially after the second post-operative week. The $\mathrm{K}$. wire was removed after 6 weeks and the cast was removed after 10 weeks. Excellent results occurred in 18 out of 24 feet operated (75\%), good in 3 feet $(12.5 \%)$, fair in 2 feet $(8.33 \%)$ and poor in one case $(4.17 \%)$. Conclusions: These modifications added more advantages to Wilson technique as a good procedure for treatment of hallux valgus.
\end{abstract}

\section{Keywords}

Hallux Valgus; Osteotomy

\section{Introduction}

Hallux valgus is not a single entity but a complex deformity of the first ray frequently accompanied by deformity and symptoms in the lesser toes [1] affecting mainly women in the fourth, fifth or sixth decade of their lives [2] [3].

The angle between the long axis of the first and second metatarsal is called intermetatarsal angle. In normal

\footnotetext{
${ }^{*}$ Corresponding author.
} 
individuals, this angle is less than 7 degrees and is regarded as pathological if greater than 10 degrees [4]. This angle increases in hallux valgus. The valgus angle of the first metatarsophalangeal joint (angle of Hallux valgus) is also increased more than 15 to 20 degrees considered the upper limits of the normal (Figure 1).

Piggott, H. (1960) [5], considered measurement of these angles to decide on suitability for operation. If the angle of hallux valgus exceeds 30 to 35 degrees, pronation of great toe usually follows. With this abnormal rotation, the abductor hallucis which is normally plantar to the axis of the first metatarsophalangeal joint moves further plantar-ward leaving the medial capsular ligament as the only supporting structure medially so, the adductor hallucis pull the great toe into valgus, then the flexor hallucis brevis, flexor hallucis longus, adductor hallucis and extensor hallucis longus increase valgus deformity of the first metatarsophalangeal joint [1].

A variety of operative procedures have been developed for the treatment of hallux valgus, [6] [7].

In the vast majority of the proposed operations, a first metatarsal osteotomy is performed in various ways and at various sites [8]-[13].

Wilson's osteotomy, first described by Wilson in 1963, is an oblique osteotomy of the first metatarsal with lateral transpositional displacement of the distal bony fragment, resulting in correction of the deformity and shortening of the first metatarsal [14]. The original concept of this osteotomy did not include any type of internal stabilization; the operation therefore frequently necessitated prolonged plaster cast immobilization due to the lack of inherent mechanical stability.

Some authors have subsequently tried various types of internal fixation of the osteotomy site, in order to obviate the need for plaster cast immobilization [15]-[17].

In the present study, we have followed the principals of Wilson technique (1963) for doing an oblique distal metatarsal osteotomy with some modifications as regard to the medial skin incision which is shifted from the dorsomedial aspect plantar-wards and the use of internal fixation of the osteotomy site by kirschner wire to fix both fragments.

\section{Material and Method}

Twenty four operations for hallux valgus were performed on fifteen patients; nine of them were operated bilaterally and six patients on one side. Ten patients were males and five females. The age of the patients ranged from 24 to 45 with a mean age of 34.5 years. The indications for the operation were pain, functional disability, cosmetic reasons, problems with shoe-fitting, and hallux valgus of 15 degrees or more with or without symptomatic bunion. The patients were evaluated clinically and radiologically.

\subsection{Pre-Operative}

\subsubsection{Clinical Assessment}

The patients were examined and the degree of hallux valgus was noticed. The range of dorsiflexion and plantar flexion of the first metatarsophalangeal joint was assessed, painful callosities on plantar surface were noted. One patient showed displacement of extensor hallucis longus laterally and plantar wards exaggerating the deformity.

\subsubsection{Radiological Assessment}

Standardized radiographs were done according to Ellis (1951) [18]. At the time of exposure each patient was

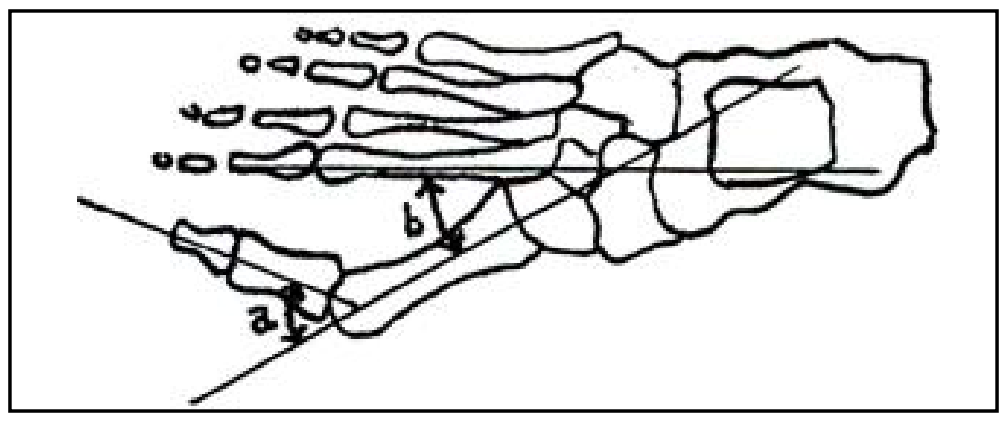

Figure 1. Hallux valgus angle (a) intermetatarsal angle (b). 
standing, bearing weight and the tube was at constant height of 36 inches above the table, the beam was directed at the midtarsal joint and angled 15 degrees posteriorly from the vertical to include the ankle joint and ensuring alignment of hind foot. The angle of hallux valgus and the intermetatarsal angle were measured.

\subsection{Operative Technique}

Under tourniquet a medial skin incision was done following the line of the first metatarsal and the proximal phalanx. This results in a concave incision curved slightly plantar wards, (instead of the conventional dorsomedial incision) after closure. The wound is deepened to expose the exostosis and the distal part of the first metatarsal. The capsular flap is raised with a base distally at the base of proximal phalanx. Removal of medial eminence followed, and then an oblique osteotomy was performed in distal part of metatarsal shaft beginning at the proximal edge of medial eminence and directed proximally and laterally at an angle of 45 degrees to the longitudinal axis of the metatarsal shaft.

Lateral and proximal displacement of the distal fragment was then done while holding the hallux in an overcorrected position. Fixation of both fragments after correction of the deformity was carried out with K. wire protruding subcutaneously. The tip of the proximal fragment should be cut off. Medial capsulorrhaphy was done and the skin was closed. A wet cotton bolus is placed between the first and second toes to preserve correction. A below the knee plaster cast was applied.

\subsection{Post Operative Care}

Post-operative radiographs were done similar to those done pre-operatively. Repeated measurements of angles as previously mentioned were performed and compared with pre-operative measurements to determine the degree of correction. Partial weight-bearing was encouraged after the second post-operative week and $k$. wire was removed after 6 weeks, then another below the knee plaster cast was applied for further 4 weeks. The plaster cast was removed and follow up X-ray was done.

\section{Results}

The patients were followed up clinically and radiologically for a period ranged from one year to two years with an average of 12 months. Evaluation of the end results was done according to criteria proposed by Butson (1980) [19] including the following parameters: appearance, comfort, range of movement and function.

\subsection{Clinical Evaluation}

Appearance: including improvement in the appearance of the foot to the extent that it is socially acceptable and the foot is not splayed, has no bunion and has natural appearing alignment of the first metatarsal and hallux relative to the rest of the foot.

Comfort: including relieve of pain on walking, no painful callus beneath the prominent first metatarsal head, no painful bunion with or without a bursa

Range of movement: including good range of movement at the first metatarsophalangeal joint.

Function: including preservation of function of the first metatarsophalangeal joint allowing normal progression from foot flat to toe-off in the gait cycle.

According to the previous factors the following grading score was recommended:

Excellent: patient had perfect appearance, comfort, range of movement and function;

Good: patient had one imperfect factor;

Fair: patient had significant complaint;

Poor: patient was dissatisfied with the results.

Excellent results occurred in 18 out of 24 feet operated (75\%), while it was good in 3 feet (12.5\%), fair in 2 feet $(8.33 \%)$ and poor in one case $(4.17 \%)$ (Table 1$)$.

In two cases graded as fair one of them was complaining from limitation of movement of metatarsophalangeal joint and the other had superficial infection which was controlled later on. In the case graded as poor one, the fixation was lost early due to pin tract infection. Extraction of K. wire was done early before consolidation and later on this patient developed metatarsalgia. 
Table 1. Clinical results.

\begin{tabular}{|c|c|c|c|}
\hline & No. of operations & Total number & $\%$ \\
\hline Excellent & 18 & 24 & $75 \%$ \\
\hline Good & 3 & 24 & $12.5 \%$ \\
\hline Fair & 2 & 24 & $8.33 \%$ \\
\hline Poor & 1 & 24 & $4.17 \%$ \\
\hline
\end{tabular}

\subsection{Radiological Assessment}

The intermetatarsal angle (the angle between first and second metatarsals) was used as a deformity index and the change in this angle was a satisfactory indicator of the improvement resulting from the operative procedure. In excellent result this angle was not more than 8 degrees. Moreover, the change in the angle of hallux valgus (the angle formed by the first metatarsal and the phalanges of the hallux) must be recorded (Table 2).

In all cases, the intermetatarsal angle post-operatively ranged from 6 - 8 degrees with a mean of 7 degrees and was considered as an excellent result.

\section{Complications}

- Pin tract infection occurred in one case leading to early extraction of $\mathrm{k}$. wire before consolidation with subsequent loss of fixation.

- Superficial infection occurred in one case.

- Metatarsalgia occurred in one case (in the case which developed pin tract infection).

- One case developed limitation of movement at metatarsophalangeal joint as compared to the pre-operative level.

- Avascular necrosis of the head of the first metatarsal was not reported in this study.

\section{Discussion}

Numerous surgical procedures have been designed to treat hallux valgus in young active patients with little or no destruction of the metatarsophalangeal joint ranging from resection arthroplasty [20] to distal first metatarsal osteotomy [21] or basal first metatarsal osteotomy [22]. McBride [23] had described a method for correction of hallux valgus by altering the balance between the forces of the adductor and abductor of great toe, approximation of metatarsals and correction of the deformity.

More than 130 surgical procedures have been described for the treatment of hallux valgus [24]. The common goals of all corrective procedures used are pain relief, permanent correction of the deformity and a biomechanically functional forefoot [25] [26].

Most of the procedures aim to remove medial exostosis and correct the hallux valgus and some also aim to correct metatarsus primus varus. The final result of these procedures is painless, well aligned first metatarsophalangeal joint, with preservation of dorsiflexion at that joint allowing normal progression from foot flat to toe-off in the gait cycle [27].

Several modifications have been made in the osteotomy techniques, in an effort to achieve adequate initial stability at the osteotomy site, by means of internal fixation, so as to allow safe mobilisation and early weight bearing [15]-[17] [28].

The preservation of dorsiflexion in the first metatarsophalangeal joint is important in women because any reduction will affect the patients' ability to wear high heeled shoes.

Radiographic assessment of the correction of hallux valgus may bear no relation to the clinical result, and in many instances it is undesirable to have the hallux corrected to the neutral position " 5 degrees of valgus" [29].

The correct postoperative alignment of the first metatarsal is entirely dependent on sufficient lateral displacement of the metatarsal head combined with slight plantar rotation of head fragment at the site of osteotomy which depend on clinical judgment, also shortening of the first metatarsal not more than three to four millimeters [30]. 
Table 2. Changes occur in inter-metatarsal angle and angle of hallux valgus.

\begin{tabular}{cccccc}
\hline & Pre-Op. (range) (degree) & Mean & Post-Op. (range) (degree) & Mean & No. of op. \\
\hline Intermetatarsal angle & $10-16$ & 12 & $6-8$ & 7 & 24 \\
Angle of hallux valgus & $15-40$ & 20 & $10-15$ & 12 & 24 \\
\hline
\end{tabular}

Oblique displacement osteotomy of the first metatarsal shaft reported by Wilson in [1963] has the advantage of simplicity of technique, excellent correction of deformity, minimal reduction of metatarsophalangeal joint motion, satisfactory narrowing of the forefoot and absence of metatarsalgia after surgery.

In our study, we have followed the same technique of Wilson osteotomy [1963] with some modifications to avoid complications of the procedure. The medial skin incision and not the dorsomedial incision was used to avoid injury to a cutaneous branch of the superficial peroneal nerve, or its adherence in scar tissue, also to avoid dissection of the skin plantar-wards to expose the capsule and bunion, preventing later haematoma formation, skin slough or delayed healing. Fixation of the osteotomy site by K. wire is easily extracted without a second operation. These previous modifications to Wilson technique offered additional advantages and produced better results.

Our results are encouraging. Excellent results occurred in 18 out of 24 feet operated (75\%), while it was good in 3 feet (12.5\%), fair in 2 feet (8.33\%) and poor in one case $(4.17 \%)$.

\section{Conclusion}

Pre-operative clinical and radiological assessment using the intermetatarsal angle as a criterion helps in proper evaluation of the cases pre-operatively and post-operatively.

Wilson technique is safe, simple and reliable in treating a patient with hallux valgus.

The present modifications of the original Wilson technique have the additional advantages of a safe exposure without damaging the cutaneous branch of superficial peroneal nerve with painless scar, and secure fixation of the osteotomy site to guard against dorsal displacement, excessive shortening and to allow early mobilization of the big toe.

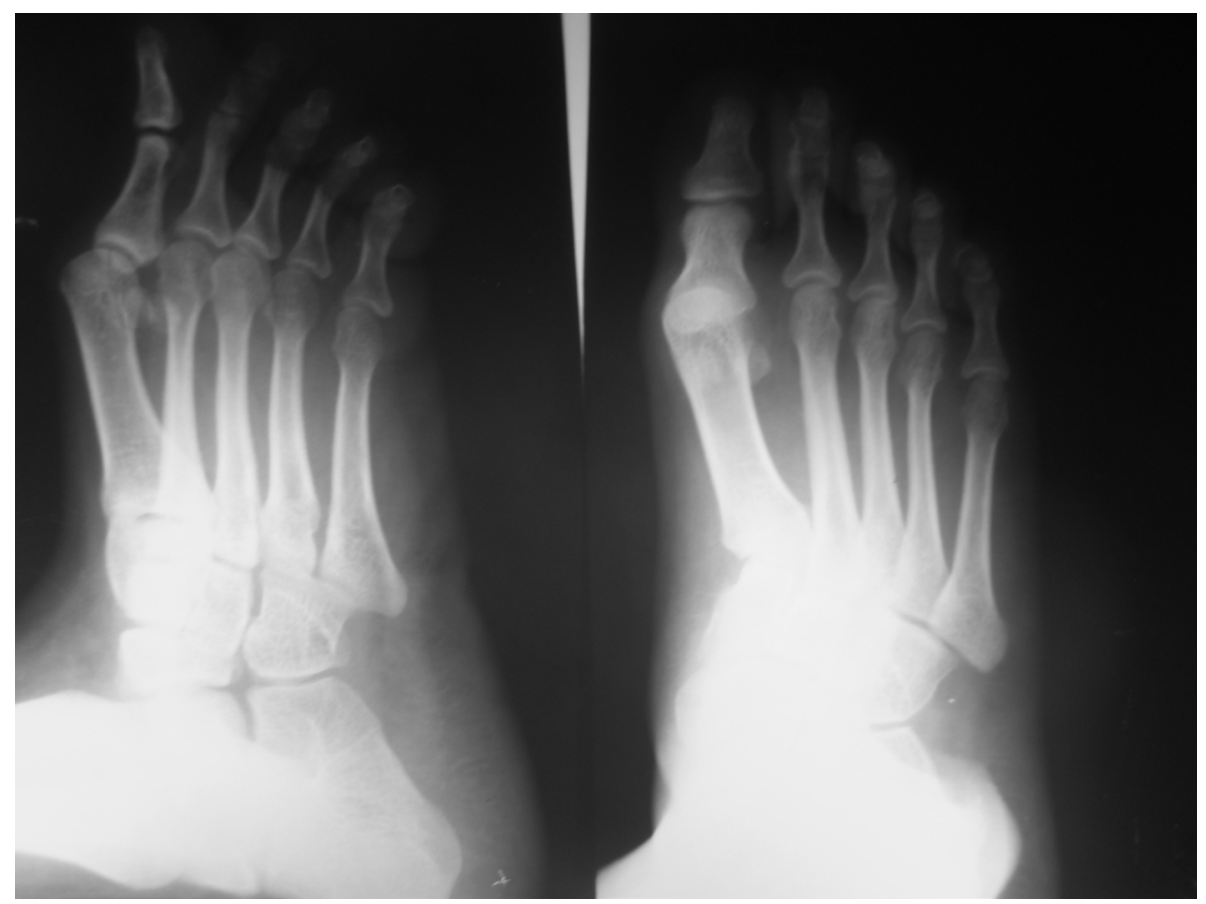

(a)

(b)

Figure 2. Preoperative X-ray. (a) oblique view (b) posteroanterior view. 


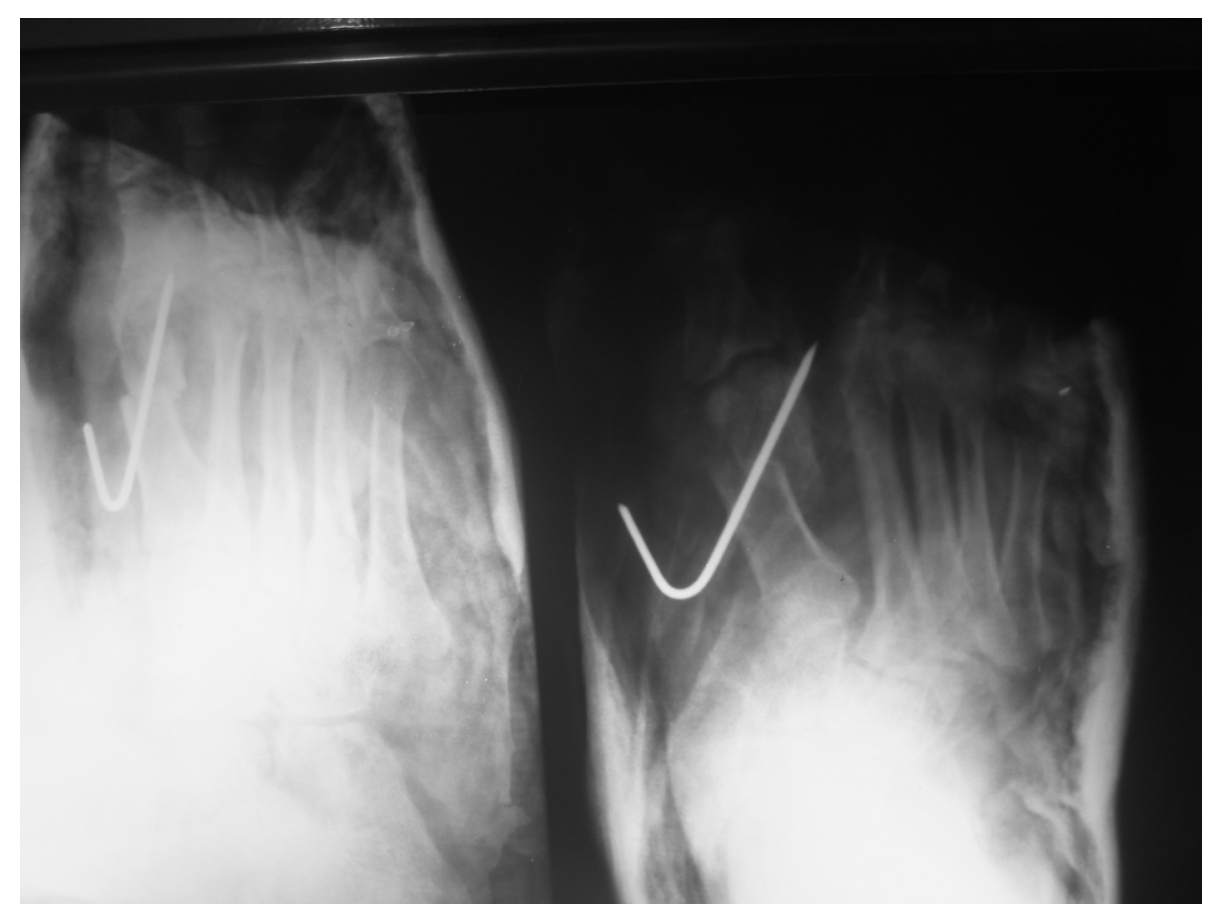

(a)

(b)

Figure 3. Postoperative X-ray. (a) oblique view (b) posteroanterior view.

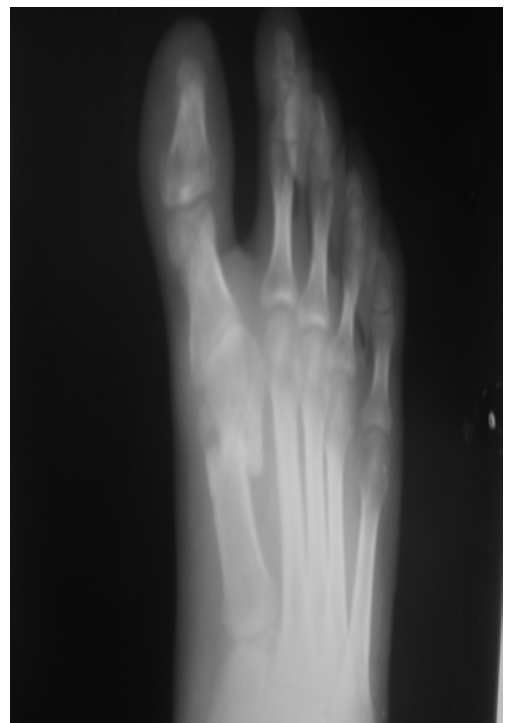

Figure 4. X-ray posteroanterior view after removal of K. wire and cast (4 months).

**A case of patient suffering from hallux valgus before operation Figure 2, postoperative X-ray Figure 3, follow up X-ray Figure 4.

\section{References}

[1] Richardson, E.G. (1987) The Foot in Adolescents and Adults. Campbell's Operative Orthopaedics, 35, 833.

[2] Coughlin, M.J. (1996) Hallux Valgus. Journal of Bone and Joint Surgery, 78-A, 932-966.

[3] Mann, R. and Coughlin, M. (1981) Hallux Valgus-Etiology, Anatomy, Treatment and Surgical Considerations. Clinical Orthopaedics, 157, 31-41. 
[4] Tachdjian, M.O. (1972) Paediatric Orthopaedics. W.B. Saunders, Philadelphia.

[5] Piggott, H. (1960) The Natural History of Hallux Valgus in Adolescence and Early Adult Life. Journal of Bone and Joint Surgery, 42-B, 749-760.

[6] Coughlin, M.J. (1996) Hallux Valgus. Journal of Bone and Joint Surgery, 78-A, 932-966.

[7] Mann, R. and Coughlin, M. (1981) Hallux Valgus-Etiology, Anatomy, Treatment and Surgical Considerations. Clinical Orthopaedics, 157, 31-41.

[8] Allen, T.R., Gross, M., Miller, J., Lowe, L.W. and Hutton, W.C. (1989) The Assessment of Adolescent Hallux Valgus before and after First Metatarsal Osteotomy. International Orthopaedics, 5, 111-115. http://dx.doi.org/10.1007/BF00267841

[9] Briggs, T.W.R., Smith, P. and McAuliffe, T.B. (1992) Mitchell’s Osteotomy Using Internal Fixation and Early Mobilisation. Journal of Bone and Joint Surgery, 74-B, 137-139.

[10] Helal, B. (1981) Surgery for Adolescent Hallux Valgus. Clinical Orthopaedics, 157, 50-63.

[11] Helal, B., Gupta, S.K. and Gojaseni, P. (1974) Surgery for Adolescent Hallux Valgus. Acta Orthopaedica Scandinavica, 45, 271-295. http://dx.doi.org/10.3109/17453677408989149

[12] Mitchell, C.L., Fleming, J.L., Allen, R., Glenney, C. and Sanford, G.A. (1958) Osteotomy-Bunionectomy for Hallux Valgus. Journal of Bone and Joint Surgery, 40-A, 41-60.

[13] Wu, K. (1992) Wu’s Bunionectomy: A Clinical Analysis of 150 Personal Cases. Journal of Foot Surgery, 31, $288-297$.

[14] Wilson, J.N. (1963) Oblique Displacement Osteotomy for Hallux Valgus. Journal of Bone and Joint Surgery, 45-B, 552-556.

[15] Allen, T.R., Gross, M., Miller, J., Lowe, L.W. and Hutton, W.C. (1989) The Assessment of Adolescent Hallux Valgus before and after First Metatarsal Osteotomy. International Orthopaedics, 5, 111-115. http://dx.doi.org/10.1007/BF00267841

[16] Geldwert, J.J., McGrath, M., Rock, G.C. and Mancuso, J.E. (1991) Wilson Bunionectomy with Internal Fixation: A Ten-Year Experience. Journal of Foot Surgery, 30, 574-579.

[17] Schemitsch, E. and Horne, G. (1989) Wilson's Osteotomy for the Treatment of Hallux Valgus. Clinical Orthopaedics, 240, 221-225.

[18] Ellis, V.H. (1951) A Method of Correcting Primus Varus. Journal of Bone and Joint Surgery, 33-B, 415-417.

[19] Butson, A.E.G. (1980) A Modification of the Lapidus Operation for Hallux Valgus. Journal of Bone and Joint Surgery, 62-B, 350-352

[20] Keller, H.L. (1904) The Surgical Treatment of Bunions and Hallux Valgus. N.Y. State. Journal of Medicine, 80, 741.

[21] Keller, J.W. (1974) Distal First Metatarsal Displacement Osteotomy. Journal of Bone and Joint Surgery, 56 A, 923.

[22] Lapidus, P.H. (1934) Operative Correction of the Metasis Varus Primus in Hallux Valgus. Surgery, Gynecology \& Obstetrics, 58, 183.

[23] Mac Bride, E.D. (1954) Hallux Valgus, Bunion Deformity, Its Treatment in (Moderate and Severe Stages). The Journal of the International College of Surgeons, 21, 99-105.

[24] Helal, B. (1981) Surgery for Adolescent Hallux Valgus. Clinical Orthopaedics, 157, 50-63.

[25] Coughlin, M.J. (1996) Hallux Valgus. Journal of Bone and Joint Surgery, 78-A, 932-966.

[26] Turnbull, T. and Grange, W. (1986) A Comparison of Keller’s Arthroplasty and Distal Metatarsal Osteotomy in the Treatment of Adult Hallux Valgus. Journal of Bone and Joint Surgery, 68-B, 132-137.

[27] Mann, R. and Hagy, J. (1979) The Function of the Toes in Walking, Jogging and Running. Clinical Orthopaedics, 142, 24.

[28] Briggs, T.W.R., Smith, P. and McAuliffe, T.B. (1992) Mitchell’s Osteotomy Using Internal Fixation and Early Mobilisation. Journal of Bone and Joint Surgery, 74-B, 137-139.

[29] Geoffrey, H., Tanzer, I. and Michael, F. (1984) Chevron Osteotomy for the Treatment of Hallux. Clinical Orthopaedics, 183, 32-36.

[30] Carr, C.R. and Boyd, B.H. (1968) Correctional Osteotomy for Metatarsus Primus Varus and Hallux Valgus. Journal of Bone and Joint Surgery, 50-A, 1353-1367. 